\title{
Desempenho agronômico de cultivares de soja na região central do Estado do Tocantins, safra 2014/2015
}

Agronomic performance of soybean cultivars in central region on Tocantins State, crop season $2014 / 2015$

\author{
F. C. Ribeiro ${ }^{1}$; G. A. Colombo ${ }^{1 *}$; P. O. S. Silva ${ }^{2}$; J. I. C. Silva ${ }^{3}$; E. A. L. Erasmo ${ }^{1}$; \\ J. M. Peluzio ${ }^{1}$

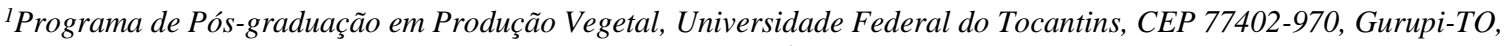 \\ Brasil \\ 2 Agro Carregal Pesquisa e Proteção de Plantas, CEP 75907-454, Rio Verde-GO, Brasil \\ ${ }^{3}$ Graduanda em Ciências Biológicas, Universidade Federal do Tocantins, CEP 77500-000, Porto Nacional-TO, Brasil
}

*colombo@uft.edu.br

(Recebido em 9 de dezembro de 2015; aceito em 16 de maio de 2016)

\begin{abstract}
Ensaios conduzidos em condições de campo são premissas básicas para gerar informações úteis à recomendação de uma cultivar em determinada situação de cultivo. Neste sentido, objetivou-se com o presente trabalho avaliar os atributos agronômicos de nove cultivares comerciais de soja na região central do estado do Tocantins, na safra 2014/2015. Foram avaliados os atributos altura de planta, altura de primeira vagem, dias para o florescimento, dias para a maturação, estande inicial de plantas, estande final de plantas, massa de mil grãos e produção de grãos. Todas as cultivares apresentaram altura de planta e primeira vagem acima do mínimo citado pela literatura como necessário para a colheita mecanizada. As cultivares SYN 13831 IPRO e SYN 1385 RR apresentaram redução no estande final de plantas, ressaltando a importância da qualidade da semente no momento de implantação da lavoura. As cultivares BMX 8579 RSF IPRO e NS 8393 RR se destacaram ao apresentarem as maiores médias de massa de mil grãos. Todas as cultivares apresentaram médias de produção de grãos acima de $3000 \mathrm{~kg} \mathrm{ha}^{-1}$.

Palavras-chave: Glycine max, Avaliação de cultivares, Melhoramento genético de plantas
\end{abstract}

Trials conducted under field conditions are basic premises to produce useful information to the recommendation of a cultivar in a particular crop situation. In this sense, the aim of the present study was to evaluate the agronomic traits of nine soybean cultivars in central region on Tocantins State, crop season 2014/2015. Were evaluated the attributes plant height, first pod insertion height, days to flowering, days to maturity, initial plant stand, final plant stand, thousand grain weight and grain yield. All cultivars have plant height and first pod insertion height above the minimum cited in the literature as necessary for mechanical harvesting. The cultivars SYN 13831 IPRO and SYN 1385 RR had reduction in the final plant stand, stressing the importance of seed quality at the time of crop establishment. The cultivars BMX 8579 RSF IPRO and NS 8393 RR have excelled by presenting the highest average mass of a thousand grains. All the cultivars present grain production averages above $3000 \mathrm{~kg} \mathrm{ha}^{-1}$.

Keywords: Glycine max, Cultivars evaluation, Breeding

\section{INTRODUÇÃO}

A soja é a oleaginosa mais cultivada no mundo e base da alimentação de diversos povos, representando importante fonte de matéria-prima para a indústria, alimentação humana e animal, sendo uma das commodities mais importantes para geração de balança comercial favorável. Possui ampla adaptação às condições brasileiras, sendo cultivada em todas as regiões do país [1].

No Estado do Tocantins, a soja é a primeira cultura em termos de participação no valor bruto da produção agrícola, sendo cultivada no período de entressafra (maio-junho) em condições de várzeas e no período de safra (novembro-dezembro), em condições de terras altas [2].

Na safra 2014/2015, a área cultivada com soja no estado foi superior a 834 mil hectares, com produção de 2,4 milhões de toneladas, e produtividade média de $2.927 \mathrm{~kg} \mathrm{ha}^{-1}$ [3]. 
A abundância de recursos hídricos, condições edafoclimáticas favoráveis, o baixo valor relativo das terras, a localização estratégica e a facilidade de acesso aos mercados são algumas das vantagens do cultivo da soja no estado do Tocantins, em relação aos outros estados produtores de soja, promovendo crescimento na área plantada e produção de grãos no estado [4]. Tais características, somadas à avaliação e introdução de novas cultivares, permitem ao estado vislumbrar posição de destaque na cadeia produtiva da soja brasileira.

A fim de atender a constante demanda por incremento em produtividade das cultivares de soja, programas de melhoramento genético buscam desenvolver genótipos superiores, resistentes aos principais estresses bióticos e abióticos que assolam a cultura [5].

A mais recente tecnologia desenvolvida e ofertada ao mercado, denominada Intacta RR2 PRO®, confere resistência ao herbicida glifosato e as principais lagartas desfolhadoras, como forma de promover maior produtividade de grãos com menor custo de produção [6].

O processo de seleção e recomendação de genótipos é considerado por muitos a etapa mais importante, onerosa e trabalhosa em um programa de melhoramento de plantas [7, 8]. Considerando que o desenvolvimento da soja é influenciado por vários fatores ambientais, entre estes a temperatura, a precipitação pluvial, a umidade relativa do ar, a umidade do solo e, principalmente o fotoperíodo [9], a avaliação do desempenho dos genótipos em diferentes locais, anos e épocas de semeadura deve ser constante, como forma de quantificar a interação genótipo $\mathrm{x}$ ambiente, e subsidiar uma recomendação ajustada para cada situação de cultivo [10].

Desse modo, objetivou-se com o presente trabalho avaliar os atributos agronômicos de nove cultivares comerciais de soja na região central do estado do Tocantins.

\section{MATERIAL E MÉTODOS}

O experimento foi conduzido no ano agrícola 2014/2015, em condições de campo na Fazenda Nossa Senhora do Carmo, situada no munícipio de Porto Nacional - TO, localizado em latitude Sul de $10^{\circ} 32^{\prime}$ 04,2' e longitude Oeste $048^{\circ} 15^{\prime}$ '50,8' de Greenwich, em altitude de 271 metros. De acordo com a classificação de Köppen o clima é do tipo B1wA'a' úmido com moderada deficiência hídrica. Os dados de precipitação pluvial e temperatura do ar observados durante a condução do experimento encontram-se na Figura 1.

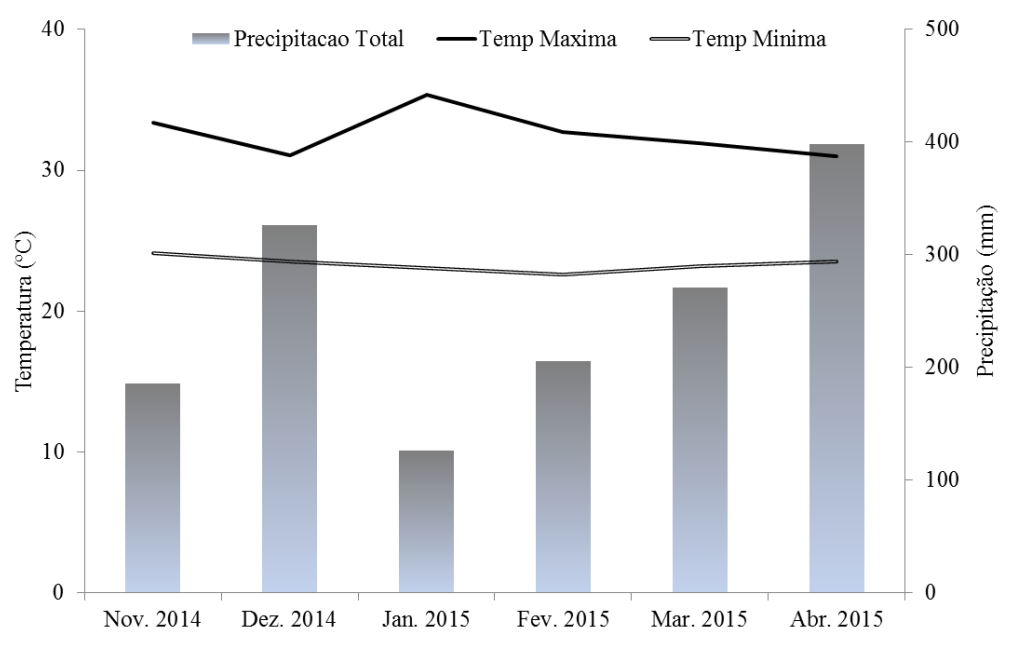

\section{Figura 1. Precipitação pluvial e temperatura mensal, observadas no decorrer da fase} experimental, em Porto Nacional-TO, safra 2014/2015.

O solo da área experimental é classificado como latossolo vermelho-amarelo distrófico [11]. Antes da instalação do experimento, foi realizada a amostragem do solo, na camada de $0-20 \mathrm{~cm}$ de profundidade, a qual apresentou a seguinte composição química: $\mathrm{pH} \mathrm{em} \mathrm{CaCl}_{2}=5,25 ; \mathrm{M} . \mathrm{O}=$ $24 \mathrm{~g} \mathrm{dm}^{-3} ; \mathrm{P}\left(\right.$ Melich $\left.^{-1}\right)=5,03 \mathrm{mg} \mathrm{dm}^{-3} ; \mathrm{Ca}=2,2 \mathrm{cmol}_{\mathrm{c}} \mathrm{dm}^{-3} ; \mathrm{Mg}=0,8 \mathrm{cmol}_{\mathrm{c}} \mathrm{dm}^{-3} ; \mathrm{H}+\mathrm{Al}=1,7$ $\mathrm{cmol}_{\mathrm{c}} \mathrm{dm}^{-3} ; \mathrm{K}=0,13 \mathrm{cmol}_{\mathrm{c}} \mathrm{dm}^{-3} ; \mathrm{CTC}=5,31 \mathrm{cmol}_{\mathrm{c}} \mathrm{dm}^{-3} ; \mathrm{V} \%=64,3$. 
O delineamento experimental utilizado foi de blocos casualizados, constituído de nove cultivares de soja, com quatro repetições. As cultivares utilizadas no experimento estão descritas na Tabela 1.

Tabela 1. Descrição das cultivares comerciais de soja utilizadas no experimento durante a safra 2014/2015. Porto Nacional - Tocantins, 2015.

\begin{tabular}{cccc}
\hline Cultivares & $\begin{array}{c}\text { Grupo de } \\
\text { Maturação }\end{array}$ & Crescimento & Cor da flor \\
\hline BMX 8579 RSF & 7.9 & Indeterminado & Roxa \\
IPRO & 8.2 & Determinado & Roxa \\
CD 2820 IPRO & 8.3 & Determinado & Branca \\
NS 8393 RR & 8.3 & Determinado & Roxa \\
MSOY 8349 IPRO & 8.3 & Indeterminado & Roxa \\
SYN 13831 IPRO & 8.4 & Semideterminado & Roxa \\
SYN 13850 IPRO & 8.5 & Indeterminado & Roxa \\
SYN 1385 RR & 8.5 & Indeterminado & Roxa \\
SYN 1387 RR & 8.5 & Determinado & Branca \\
TMG 132 RR &
\end{tabular}

Fonte: Registro Nacional de Cultivares [12].

As parcelas experimentais foram compostas de oito linhas de seis metros de comprimento, com espaçamento de 0,50 m nas entrelinhas. Na colheita, desprezaram-se as duas linhas laterais e 0,50 $\mathrm{m}$ em cada extremidade das quatro linhas centrais. A área útil da parcela foi composta pelas quatro fileiras centrais, que totalizaram área de $10 \mathrm{~m}^{2}$.

A adubação de plantio foi de $424 \mathrm{~kg} \mathrm{ha}^{-1}$ do adubo químico formulado 05-25-15, sendo realizada ainda adubação em cobertura no estádio vegetativo $\mathrm{V} 4$ com $52 \mathrm{~kg} \mathrm{ha}^{-1}$ de $\mathrm{K}_{2} \mathrm{O}$, utilizando como fonte cloreto de potássio. Por ocasião do plantio inoculou-se as sementes com estirpes de Bradyrhizobium japonicum, com a finalidade de se obter boa nodulação das raízes, garantindo o suprimento de nitrogênio à cultura.

A semeadura foi realizada 11 de novembro de 2014 com uma densidade de semeadura de 18 sementes por metro linear para cada uma das cultivares utilizadas no experimento. O controle de pragas, doenças e plantas daninhas foram realizados à medida que se fizeram necessários, conforme Sediyama [13].

Com base na área útil da parcela, foram avaliados os seguintes atributos agronômicos das cultivares: Altura da planta (AP) - distância em centímetros, medida a partir da superfície do solo até a extremidade da haste principal da planta, na época da maturação, em 10 plantas da área útil; Altura de inserção da primeira vagem (APV) - distância em centímetros, medida a partir da superfície do solo até a inserção da primeira vargem; Número de dias para o florescimento (NDF) - número de dias contados, a partir da emergência, necessários para que se tenha uma flor aberta em 50\% das plantas da parcela; Número de dias para a maturação (NDM) - número de dias contados, a partir da emergência, necessários para que se tenha $95 \%$ de vagens maduras na parcela; Estande inicial de plantas (EI) - obtido através da contagem das plantas presente em 1 metro nas linhas centrais de cada parcela, 10 dias após a emergência da cultura; Estande final de plantas $(\mathrm{EF})$ - obtido por meio da contagem das plantas presente em um metro nas linhas centrais de cada parcela, na época da maturação.

As plantas de cada parcela foram colhidas e trilhadas no estádio reprodutivo R8, ou seja, quando apresentaram o mínimo de $95 \%$ das vagens maduras. Na ocasião da colheita, avaliou-se a massa de 1000 grãos (MMG) - determinada com a pesagem de duas amostras de 1000 grãos por parcela, após a correção da umidade para 13\%, e a produtividade de grãos (PROD) - obtida por meio da massa correspondente a cada parcela, convertida em $\mathrm{kg} \mathrm{ha}^{-1}$, após correção da umidade para $13 \%$.

Os dados foram submetidos à análise de variância. As médias foram agrupadas pelo teste de Scott \& Knott a 5\% de probabilidade pelo software computacional Sisvar 5.3 [14]. 


\section{RESULTADOS E DISCUSSÃO}

Foram detectadas diferenças significativas entre as cultivares para EI e MMG $(p \leq 0,05)$, bem como para os demais atributos avaliados $(\mathrm{p} \leq 0,01)$, de forma a evidenciar o comportamento distinto entre os genótipos (Tabela 2). O coeficiente de variação para as características ficaram compreendidos entre 1,71 a 10,63 \%, indicando alta precisão e homogeneidade das condições experimentais, segundo parâmetro estabelecido por Pimentel-Gomes [15].

Tabela 2. Resumo da análise de variância dos atributos altura de planta (AP), altura da primeira vagem $(A P V)$, número de dias para o florescimento $(N D F)$, número de dias para a maturação (NDM), estande inicial das plantas (EI), estande final das plantas (EF), massa de

1000 grãos $(M M G)$ e produtividade de grãos (PROD) de nove cultivares de soja, safra 2014/2015. Porto Nacional-Tocantins, 2015.

\begin{tabular}{cccccccccc}
\hline Fonte de & & \multicolumn{7}{c}{ QUADRADO MÉDIO } \\
\cline { 2 - 10 } Variação & GL & AP & APV & NDF & NDM & EI & EF & MMG & PROD \\
\hline Trat & 8 & $2283,57^{* *}$ & $15,20^{* *}$ & $15,44^{* *}$ & $38,11^{* *}$ & $1,90^{*}$ & $9,13^{* *}$ & $1212,39^{*}$ & $335548^{*}$ \\
Bloc & 3 & $17,05^{* *}$ & $0,96^{\text {ns }}$ & $15,43^{* *}$ & $2,34^{\text {ns }}$ & $1,10^{\text {ns }}$ & $7,08^{*}$ & $1042,13^{*}$ & $261175^{\text {ns }}$ \\
Resíd & 24 & 2,22 & 0,73 & 1,15 & 1,79 & 0,55 & 2,23 & 181,71 & 93737 \\
\hline C.V.\% & 1,71 & 5,46 & 3,08 & 1,16 & 4,60 & 10,63 & 8,00 & 8,24 \\
\hline
\end{tabular}

${ }^{\text {ns }}$ não significativo; ${ }^{* *}$ significativo a $1 \%$ de probabilidade e ${ }^{*}$ significativo a $5 \%$ de probabilidade pelo teste F.

Com relação à altura de plantas (Tabela 3), a cultivar SYN 13831 IPRO apresentou a maior média de altura de plantas $(129,68 \mathrm{~cm})$, diferindo estatisticamente das demais cultivares. Contrariamente, a cultivar SYN 13850 IPRO apresentou altura média de 53,13 cm, estatisticamente menor que as demais. Segundo Barros et al. (2003) [7], a altura de plantas ideal deve ser no mínimo $50 \mathrm{~cm}$, afim de evitar perdas durante o processo de colheita mecanizada. Neste sentido, plantas mais altas devem ser privilegiadas por apresentarem efeitos diretos sobre a produção grãos [16].

Tabela 3. Valores médios de altura de plantas (AP), altura de inserção de primeira vagem (AIPV), número de dias para o florescimento (NDF) e número de dias para a maturação (NDM) de nove cultivares de soja conduzidas na safra 2014/2015, Porto Nacional-Tocantins, 2015.

\begin{tabular}{ccccc}
\hline Cultivares & AP $(\mathbf{c m})$ & APV $(\mathbf{c m})$ & NDF (dias) & NDM (dias) \\
\hline BMX 8579 RSF IPRO & $101,88 \mathrm{c}$ & $18,58 \mathrm{a}$ & $32,00 \mathrm{~b}$ & $110,00 \mathrm{c}$ \\
CD 2820 IPRO & $68,58 \mathrm{~g}$ & $15,33 \mathrm{c}$ & $33,00 \mathrm{~b}$ & $114,00 \mathrm{~b}$ \\
NS 8393 RR & $90,53 \mathrm{e}$ & $15,35 \mathrm{c}$ & $38,00 \mathrm{a}$ & $119,00 \mathrm{a}$ \\
MSOY 8349 IPRO & $72,83 \mathrm{f}$ & $15,03 \mathrm{c}$ & $36,00 \mathrm{a}$ & $115,00 \mathrm{~b}$ \\
SYN 13831 IPRO & $129,68 \mathrm{a}$ & $16,75 \mathrm{~b}$ & $36,00 \mathrm{a}$ & $115,00 \mathrm{~b}$ \\
SYN 13850 IPRO & $53,13 \mathrm{~h}$ & $12,30 \mathrm{~d}$ & $33,00 \mathrm{~b}$ & $119,00 \mathrm{a}$ \\
SYN 1385 RR & $94,75 \mathrm{~d}$ & $13,50 \mathrm{~d}$ & $34,00 \mathrm{~b}$ & $113,00 \mathrm{~b}$ \\
SYN 1387 RR & $105,33 \mathrm{~b}$ & $17,70 \mathrm{a}$ & $36,00 \mathrm{a}$ & $115,00 \mathrm{~b}$ \\
TMG 132 RR & $66,30 \mathrm{~g}$ & $15,80 \mathrm{c}$ & $36,00 \mathrm{a}$ & $119,00 \mathrm{a}$ \\
\hline Médias & 86,99 & 15,59 & 34,89 & 115,44 \\
\hline
\end{tabular}

Médias seguidas pela mesma letra na coluna pertencem ao mesmo grupo estatístico ao nível de 5\% de probabilidade, pelo teste de Scott \& Knott.

Quanto à altura de inserção de primeira vagem (APV), todas as cultivares apresentaram valores médios acima $12 \mathrm{~cm}$, definido por Sediyama et al. (2009) [13] como o mínimo para evitar perdas na colheita mecanizada. As maiores APV foram observadas nas cultivares BMX 8579 RSF IPRO $(18,58 \mathrm{~cm})$ e SYN 1387 RR $(17,70 \mathrm{~cm})$ diferindo-se estatisticamente das demais cultivares. As menores APV foram observadas nas cultivares SYN 13850 IPRO $(12,30 \mathrm{~cm})$ e SYN 1385 RR $(13,50 \mathrm{~cm})$. Ressalta-se, contudo, que a seleção de plantas com baixa altura de 
inserção de primeira vagem poderá acarretar em perdas na colheita mecanizada de modo que, inviabilize o cultivo das cultivares que apresentem essa característica agronômica [4].

Em relação ao número de dias para o florescimento (NDF), a cultivar NS 8393 RR (38,00 dias), seguido das cultivares TMG 132 RR, MSOY 8349 IPRO, SYN 1387 RR e SYN 13831 IPRO (36,00 dias) formaram grupo superior, estatisticamente distinto das demais. Em contrapartida, as cultivares BMX 8579 RSF IPRO (32,00 dias), CD 2820 IPRO (33,00 dias), SYN 13850 IPRO (33 dias) e SYN 1385 RR (34 dias) apresentaram as menores médias de NDF. Esses resultados seguem uma tendência descrita por Barros et al. (2003) [7] e Peluzio et al. (2005) [17], que, em trabalhos conduzidos no cerrado tocantinense, observaram variações relativamente pequenas quanto ao número de dias necessários à florescimento das cultivares.

A cultivar BMX 8579 RSF IPRO também se destacou ao apresentar a menor média de numero de dias para maturação (NDM), com 110 dias, estatisticamente menor que as demais. Tal comportamento, somado ao fato desta cultivar ter apresentado menor NDF, evidencia a precocidade do genótipo, quando comparado com as demais cultivares avaliadas.

Os atributos NDF e NDM, em virtude da sensibilidade fotoperiódica da cultura, são importantes critérios para recomendação do cultivar, de modo a favorecer o escalonamento da semeadura e colheita, de acordo com as condições climáticas da região. Tal escalonamento é realizado no intuito de minimizar a chance de ocorrência de períodos prolongados de estresses hídricos nas fases mais críticas de desenvolvimento da planta, e de excesso hídrico próximo à colheita [4].

Quanto ao estande inicial de plantas (EI), a cultivar SYN 1387 RR apresentou maior densidade de plantas na linha de semeadura $\left(17,13 \mathrm{pl} \mathrm{m}^{-1}\right)$ diferenciando-se estatisticamente das demais (Tabela 4). Diferentemente, as cultivares SYN 13831 IPRO (15,00 $\left.\mathrm{pl} \mathrm{m}^{-1}\right)$ e SYN 1385 RR (15,38 $\mathrm{pl} \mathrm{m}^{-1}$ ) formaram grupo estatístico com as menores médias de EI. Inicialmente, Embrapa (2005) [18] indicava como padrão uma população de 18 plantas $\mathrm{m}^{-1}$ linear, número que foi reduzido gradativamente até 15 plantas $\mathrm{m}^{-1}$ linear. Contudo, Neves et al. (2013) [19] afirmam que esse atributo pode variar em função do cultivar, do regime pluviométrico no momento de implantação da cultura, e época de semeadura. Vasquez et al. (2008) [20], analisando os efeitos da redução da população de plantas sobre alguns atributos da cultura da soja, relataram que a cultura é capaz de suportar grandes reduções da população de plantas sem perdas significativas de produtividade de grãos, e que essa capacidade depende da cultivar, suportando até $45 \%$ de redução.

Tabela 4. Valores médios de estande inicial de plantas (EI), estande final de plantas (EF), massa de 1000 grãos (MMG) e produtividade de grãos (PROD) de nove cultivares de soja conduzidas na safra 2014/2015, Porto Nacional - Tocantins, 2015.

\begin{tabular}{|c|c|c|c|c|}
\hline Cultivares & EI $\left(p l\right.$ m $\left.^{-1}\right)$ & EF $\left(\mathbf{p l ~ ~ m ^ { - 1 } )}\right.$ & MMG (g) & PROD $\left(\mathrm{kg} \mathrm{ha}^{-1}\right)$ \\
\hline BMX 8579 RSF IPRO & $15,43 \mathrm{c}$ & $14,50 \mathrm{a}$ & $198,77 \mathrm{a}$ & $3790 \mathrm{a}$ \\
\hline CD 2820 IPRO & $16,08 \mathrm{~b}$ & $14,38 \mathrm{a}$ & $167,05 \mathrm{~b}$ & $4236 \mathrm{a}$ \\
\hline NS 8393 RR & $16,45 \mathrm{~b}$ & $14,00 \mathrm{a}$ & $185,79 \mathrm{a}$ & $3846 \mathrm{a}$ \\
\hline MSOY 8349 IPRO & $16,63 \mathrm{~b}$ & $14,50 \mathrm{a}$ & $167,99 \mathrm{~b}$ & $3902 \mathrm{a}$ \\
\hline SYN 13831 IPRO & $15,00 \mathrm{c}$ & $11,50 \mathrm{~b}$ & $156,89 \mathrm{~b}$ & $3251 \mathrm{~b}$ \\
\hline SYN 13850 IPRO & $16,50 \mathrm{~b}$ & $14,50 \mathrm{a}$ & $169,73 \mathrm{~b}$ & $3608 \mathrm{~b}$ \\
\hline SYN 1385 RR & $15,38 \mathrm{c}$ & $11,75 \mathrm{~b}$ & $169,60 \mathrm{~b}$ & $3464 \mathrm{~b}$ \\
\hline SYN 1387 RR & $17,13 \mathrm{a}$ & $16,25 \mathrm{a}$ & $164,55 \mathrm{~b}$ & $3520 \mathrm{~b}$ \\
\hline TMG 132 RR & $16,00 \mathrm{~b}$ & $15,00 \mathrm{a}$ & $136,04 \mathrm{c}$ & $3834 \mathrm{a}$ \\
\hline Médias & 16,06 & 14,04 & 168,49 & 3717 \\
\hline
\end{tabular}

Médias seguidas pela mesma letra na coluna pertencem ao mesmo grupo estatístico ao nível de $5 \%$ de probabilidade, pelo teste de Scott \& Knott.

As cultivares SYN 13831 IPRO e SYN 1385 RR destacaram-se por apresentar as menores médias de estande final (EF), com $11,50 \mathrm{pl} \mathrm{m}^{-1}$ e $11,75 \mathrm{pl} \mathrm{m}^{-1}$, respectivamente. Chama-se atenção ao fato de tais cultivares também terem apresentado as menores médias de EI. Essa observação ressalta a importância da qualidade da semente no momento de implantação da 
lavoura. Segundo Teófilo et al. (2007) [21], sementes com germinação e vigor reduzidos originam lavouras com população desuniforme e, consequentemente, acarretam sérios prejuízos econômicos. A correta distribuição espacial de plantas está diretamente relacionada com o aumento em produtividade, ao favorecer o melhor aproveitamento da luminosidade, água e nutrientes [22].

Em relação à massa de 1000 grãos (MMG), as cultivares BMX 8579 RSF IPRO e NS 8393 RR apresentaram as maiores médias (198,77 e 185,79 gramas, respectivamente), diferenciando estatisticamente das demais cultivares. A MMG é um atributo determinado geneticamente, porém, fortemente influenciado pelo ambiente [23]. Os valores de MMG observados no presente trabalho estão acima dos encontrados por Peluzio et al. (2002) [24], em ensaios conduzidos no cerrado tocantinense. Tal fato evidencia o aprimoramento deste atributo nas cultivares modernas, muito em função de estar diretamente relacionado à produção de grãos [10].

Quanto a produtividade de grãos (PROD), as cultivares CD 2820 IPRO (4236 kg ha-1), MSOY 8349 IPRO (3902 kg ha' ${ }^{-1}$, NS 8393 RR (3846 kg ha-1), TMG 132 RR (3834 kg ha-1) e BMX 8579 RSF IPRO (3790 kg ha $\left.{ }^{-1}\right)$ formaram grupo estatístico com as maiores médias. No presente trabalho, todos os genótipos apresentaram médias de PROD acima de $3000 \mathrm{~kg} \mathrm{ha}^{-1}$. Richetti (2012) [25], estudando a viabilidade econômica na cultura da soja transgênica, obteve produtividade de equilíbrio a partir de $2448 \mathrm{~kg} \mathrm{ha}^{-1}$. Mesmo considerando que o retorno econômico da soja está em função de sua cotação diária, as cultivares avaliadas apresentaram desempenho produtivo satisfatório, representando interessante alternativa para cultivo em condições de cerrado tocantinense.

\section{CONCLUSÃo}

A altura de planta e de inserção de primeira vagem das cultivares de soja avaliadas são adequadas à colheita mecanizada.

As cultivares BMX 8579 RSF IPRO e NS 8393 RR se destacam quanto a massa de mil grãos acima de 185 gramas.

Todas as cultivares são promissoras e adaptadas às condições edafoclimáticas de Porto Nacional - TO, com produtividade de grãos superior a $3000 \mathrm{~kg} \mathrm{ha}^{-1}$.

\section{AGRADECIMENTOS}

À Prince Consultoria e Pesquisa Agronômica pelo apoio financeiro.

\section{REFERÊNCIAS BIBLIOGRÁFICAS}

1. Venturoso LR, Caron BO, Schimidt D, Bergamin AC, Valadão Júnior DD, Jakelaitis A. Efeito da época de semeadura sobre caracteres agronômicos em cultivares de soja em Rolim de Moura - RO. Bioscience Journal. 2009; 25(4):73-81.

2. Almeida RD, Peluzio JM, Afférri FS. Divergência genética entre cultivares de soja, sob condições de várzea irrigada, no sul do Estado Tocantins. Revista Ciência Agronômica. 2011;42(1):108-115.

3. Companhia Nacional de Abastecimento. Acompanhamento da safra brasileira: grãos safra 2014/2015 - décimo primeiro levantamento. [Internet] Brasília: CONAB; [citado em 2015 Sep 25]. Disponível em: http://www.conab.gov.br/OlalaCMS/uploads/arquivos/15_08_18_10_30_18_boletim_graos_ agosto_2015.pdf

4. Peluzio JM, Fidelis RR, Almeida D, Barbosa VS, Richter LHM, Silva RR, Afférri FS. Desempenho de cultivares de soja, em duas épocas de semeadura, no sul do Estado de Tocantins. Bioscience Journal. 2006; 22(2):69-74.

5. Vasconcelos ES, Reis MS, Sediyama T, Cruz CD. Produtividade de grãos, adaptabilidade e estabilidade de genótipos de soja de ciclos precoce e médio. Semina: Ciências Agrárias. 2015;36(3):1203-1214, doi: 10.5433/1679-0359.2015v36n3p1203.

6. Mateus RPG, Silva CM. Avanços biotecnológicos na cultura da soja. Campo Digital. 2013;8(2):23-27. 
7. Barros HB, Peluzio JM, Santos MM, Brito EL, Almeida RD. Efeitos das épocas de semeadura no comportamento de cultivares de soja, no sul do Estado do Tocantins. Revista Ceres. 2003;50(291):565-572.

8. Pires LPM, Peluzio JM, Cancellier LL, Ribeiro GR, Colombo GA, Afférri FS. Desempenho de genótipos de soja, cultivados na região centro-sul do estado do Tocantins, safra 2009/2010. Bioscience Journal. 2012;28(2):214-223.

9. Rocha RS, Da Silva JAL, Neves JA, Sediyama T, Cássia Teixeira R. Desempenho agronômico de variedades e linhagens de soja em condições de baixa latitude em Teresina-PI. Revista Ciência Agronômica. 2012;43(1):154-162.

10. Sediyama T, Teixeira RC, Reis, MS. Melhoramento da Soja. In: Borem A. Melhoramento de espécies cultivadas. Viçosa: UFV, 2005. p. 553-602.

11. Empresa Brasileira de Pesquisa Agropecuária - EMBRAPA. Sistema brasileiro de classificação de solos. 3 ed. Rio de Janeiro: EMBRAPA/CNPSO, 2013. 353 p.

12. BRASIL. Ministério da Agricultura, Pecuária e Abastecimento. Registro Nacional de Cultivares RNC. Disponível em: 〈http://www.agricultura.gov.br/>. Acesso em: 10 março 2016.

13. Sediyama, T. Tecnologias de produção e usos da soja. Viçosa. Editora Mecenas, 2009, 314 p.

14. Ferreira DF. Sisvar: a computer statistical analysis system. Ciência e Agrotecnologia. 2011;35(6):1039-1042.

15. Pimentel-Gomes, F. Curso de estatística experimental. 14. ed. Piracicaba: Livraria Nobel, 2000.

16. Fidelis RR, Peluzio JM, Pinto LC, Carvalho GL, Nascimento IR, Rodrigues AM. Desempenho de cultivares de soja semeadas sob pastagens degradadas. Ambiência. 2011;7(1):123-131, doi: 10.5777/ambiencia.2011.01.01rc

17. Peluzio JM, Almeida D, Francisco ER, Fidelis RR, Richter LHM, Barbosa VS. Comportamento de cultivares de soja no sul do estado do Tocantins. Bioscience Journal. 2005;21(3):113-118.

18. Empresa Brasileira de Pesquisa Agropecuária - EMBRAPA. Tecnologias de produção de soja Região Central do Brasil 2004. Londrina: EMBRAPA SOJA, 2005. 237 p.

19. Neves JA, Silva JAL, Rafael D, Barbosa S, Sediyama T, Cássia Teixeira R, Rocha RS, Agronomic performance of soybean genotypes in low latitude in Teresina-PI, Brazil. Journal of Agricultural Science. 2013;5(3):243-253, doi: 10.5539/jas.v5n3p243

20. Vasquez GH, Carvalho NM, Borba MMZ. Redução na população de plantas sobre a produtividade e a qualidade fisiológica da semente de soja. Revista Brasileira de Sementes. 2008;30(2):1-11, doi: 10.1590/S0101-31222008000200001

21. Teófilo EM, Dutra AS, Dias FTCA. Potencial Fisiológico de Sementes de Soja produzidas no Estado do Ceará. Revista Ciência Agronômica. 2007;38(4):401- 406.

22. Silva WB, Petter FA, De Lima LB, Andrade FR. Desenvolvimento inicial de Urochloa ruziziensis e desempenho agronômico da soja em diferentes arranjos espaciais no cerrado Mato-Grossense. Bragantia. 2013;72(2):146-153, doi: 10.1590/S0006-87052013000200006

23. Navarro Junior HM, Costa JA. Contribuição relativa dos componentes do rendimento para produção de grãos em soja. Pesquisa Agropecuária Brasileira. 2002;37(3):269-274, doi: 10.1590/S0100204X2002000300006

24. Peluzio, J.M.; Barros HB, Santos MM, Reis MSR. Comportamento de duas cultivares de soja em diferentes populações de plantas sob condições de várzea irrigada no sul do estado do Tocantins. Revista Agricultura Tropical. 2002;6(6):69-80.

25. Richetti A. Viabilidade econômica da cultura da soja na safra 2012/2013, em Mato Grosso do Sul. Dourados: Embrapa, 2012. (Comunicado técnico, 177). 